Defining Ecosystemic Agency in Live Performance. The Machine Milieu Project as Practice-Based Research.

by Agostino Di Scipio and Dario Sanfilippo

\section{Premises and overview}

In live performance setups, computing resources typically represent powerful yet subordinated technical agencies, piloted by practitioners or implementing well-planned automations and compositional algorithms. The necessary analog equipment is viewed, to a large extent, as a neutral chain of electroacoustic transductions, transparently channeling and amplifying input and output sound signals. The local physical environment is itself either ignored or "tuned out," implicitly understood as irrelevant to the performance process and to the actual sound events it brings forth.

In a different perspective, the complete performance framework can be understood, instead, as an integral "performance ecosystem" [Waters 2007, 2013], and the whole field of interactions between human performer(s), equipment (computer devices and analog electroacoustics) and the surrounding environment can be addressed in its terms and turned into a creative medium. In earlier experiences, the authors of this paper have independently designed their performance ecosystems as complex dynamical systems to be creatively explored in live performance and sound installations contexts [Di Scipio 2003, 2008; Sanfilippo 2013, 2018]. In 2014 they started a collaborative effort, which was eventually given the project title Machine Milieu.

The idea is to consider the human performer, equipment, and performance space as three sites of agency mutually connected in the medium of sound, capable of developing an integral and possibly autonomous performance ecosystem based on site-specific sonic information only ("sound is the interface" [Di Scipio 2003]). Central is the notion that the computer-implemented processes involved may somehow "make sense" of what happens sound-wise in the local, shared environment, and act accordingly.

This effort points to a situated, and hybrid process whose system dynamics, whose musical identity, or Self, develops from its structural coupling with and exposure to an array of external forces and agencies (i.e., to several "non-Selves," or "other 
Selves") present in the local environment [Di Scipio 2011]. We lean on Maturana \& Varela's [1980] well-known discussion of autopoiesis in living systems: an autopoietic agent is a system that develops its Self as its component parts work together and construe a whole by making something in and to the environment to which they are structurally coupled, while allowing the environment to bias their operations. Our idea is to implement an autopoietic dynamics able to determine the emergence of consistent behavior in sound. While the interacting parts bring forth a whole, the whole in turn biases or bends the individual parts in their further doing and thus reinforce the ensemble in its distinct dynamical behavior. Both "upward" and "downward" causations are involved in the unfolding of complex dynamical systems [Benkirane et al. 2002].

Such kind of agency can be likened to a minimally cognitive system [Etxeberria et al. 1994, Barandirian et al. 2006], i.e., an entity that construes information about its surrounding in order to establish a positive and indeed constructive relationship with it. In Maturana and Varela [1980], living systems are understood as cognitive systems. Crucial here is the Batesonian definition of information as something built and processed by a system coupled to an environment [Bateson 1972]. The inevitable difference between information construed by the system for itself, and what "really" is out there, is what keeps the system process going. For Bateson, a bit of information is notoriously defined as a "difference which makes a difference": a differential quantum that travels and spreads across the circuit and undergoes a process of recursive interactions and transformations. Information and computation in a cognitive agent were defined by Heinz von Foerster [2007] as recursive processes in a system having sufficient complexity in dealing with the environment. This relates also to Robert Ashby's notion of a minimum of requisite variety [1958] necessary in order for a system to be capable of self-regulation.

In Machine Milieu, we design a bundle of recursive processes which shape an overall ecosystemic ensemble with no central site of agency, no unilateral control over the ensemble or any of the single parts. Each manifestation of such an assemblage is specified by a set of interdependent processes and variables. That results in a complex dynamical system 
[Benkirane et al. 2011, Mitchell 2009] where agency at the level of the individual parts is negotiated and mediated with agency at the level of the emerging whole. Changing the conditions of operations (physical environment, analog equipment, and the runtime variables of computer-implemented processes) creates a different system with its own set of evolving ecosystemic dynamics, revealing different potentials emerging from the same structural design. Many repeated explorations of such a performance ecosystem are required in order for its sonic potential to be assessed.

We may eventually call music the traces of such process in sound. By and large, music is always something that happens in a well-balanced triangular interaction of humans, tools, and places.

Autonomy and feedback in live performance

Defining autonomy in music systems is a difficult task [Bown \& Martin 2012]. In bio-cybernetic terms, a system can be called an autonomous agency to the extent that it regulates itself by observing the traces it leaves in the environment. It is both open and closed to the environment (hence, to other agencies inhabiting the environment): it eventually "closes onto itself" through the environment to which it is open [Maturana \& Varela 1980, Clarke \& Hansen 2009]. A fruitful notion of autonomy in music-related systems, then, would include this operational loop through an environment hosting a variety of sources of sonic information. In other words, a system's autonomy requires a level of heteronomy, born of heterogeneous forces situated in and mediated by the sound environment. We attribute ecosystemic agency to a system whose composite process does not only "deemphasize [...] the categorial split between humans and machines" [Rutz 2016b] but also relativizes or waives the split between the human-andmachine couple and the environment.

This notion of autonomous agency implies a qualified notion of interaction, understood as the "mutual influence" of two or more structurally coupled entities. In a performance ecosystem, both humans and machines should be acknowledged autonomous behavior to the extent that they can act (sound-wise) in the environment while also changing their actions upon contact with other 
agencies in the environment. Feedback should be seen as a structural feature.

Clearly, "autonomous" is not to be confused with "automated." Automation, implies centralized control. In typical computer music designs, sound events are "automatically" scheduled, or driven, by some formal rules (either a deterministic or indeterministic process), which shape the musical flow in a domain entirely independent of - and fundamentally (in)different to - the medium of sound (be it understood as signal or as a physical and perceptual phenomenon). In our design, leaning as much as possible on the experiential milieu of sound, we develop larger musical articulations out of the material acoustical environment and its "background noise." Viewing the performance ecosystem as an autonomous agency developing itself based on situated acoustical events can be a significant shift in substantiating an operative metaphor of the "living," as evoked in live (living) performance practice as well as in the live (lived) experience of sound and music [Di Scipio, forthcoming].

The implementation of multiple feedback delay networks (FDN) is a central factor in our practice, for the peculiar dynamical characteristics they exhibit seem well suited to human-machine interactions in the context of music performance [Sanfilippo \& Valle 2013]. In our strategy, even the slightest "differences" in the medium enter into the system, whether originating from performers' actions, ambience noise, or machine output, and are then circulated, and modulated across a network of feedback mechanisms. When the differences are truly informative (in Batesonian terms), a larger process is triggered, resulting in short-term and long-term variations, at both the smallest time scale (signal contours and related timbral percepts), and the general unfolding and behavioral transitions in the systems.

Description of the MACHINE MILIEU project

General infrastructure

A sketch of the Machine Milieu infrastructure is seen in Figure 1. It includes two performers with their Computer Units, microphones, and loudspeakers, to be placed at strategic positions in the performance space (Environment). Bold lines stand for audio signal flows, dashed lines for control signals. Note that several feed- 
back paths can be traced across the complete infrastructure.

The signal processing in the two Computer Units is made dependent on both the sonic context, as captured through microphones and internal analysis, and the performers' direct access to relevant variables in the processing algorithms. Since performers typically act depending on what they hear in the environment, by delivering sound at specific positions in the performance space, the loudspeakers act not just as endpoints of the sound-generating system, but as means to elicit the space's acoustical response, which will, in turn, affect the computer processes (via the microphones).

Performers can be committed to acting not only on the computer variables, but also on loudspeakers and microphones (e.g., changing their position or altering the acoustical context in other ways, causing acoustical shadows and other mechanical effects in the sound diffusion). They can hardly know beforehand the longterm consequences of their actions, but they will eventually face them and feel the necessity to mitigate any undesirable drifts. Incurring a loss of control into "drifts" is not inappropriate, as it may "stress" the system to

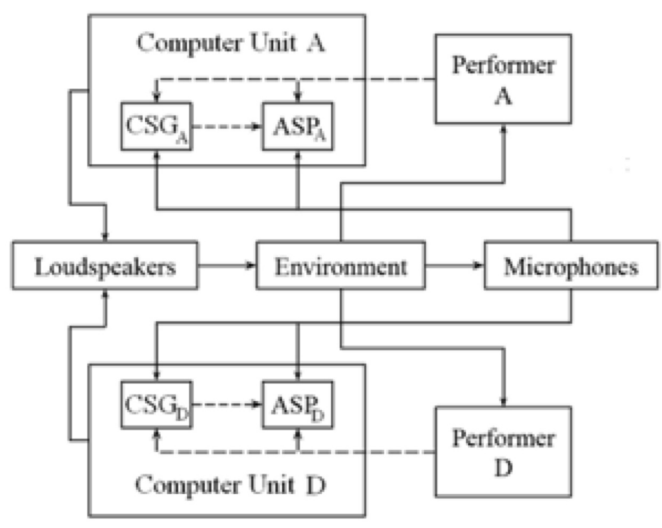

Figure 1. Generic layout of the Machine Milieu infrastructure.

operate in boundary conditions which reveal otherwise unattainable behaviors. One should handle such situations cautiously, making sure the process does not get stuck at the boundaries of its inherent system dynamics, eventually resuming more viable working conditions.

\section{Technical aspects}

At the present stage, all digital signal processing algorithms in Machine Milieu are implemented with Pure Data Vanilla and with Kyma/Pacarana. The bulk of it consists of time-variant FDNs though which several nonlinear signal transformations are intermingled. Several positive and negative 
feedback mechanisms are included, meant to counterbalance local exchanges in the network and to create a larger variety in the resultant sound fabric. Feedback mechanisms are also used to implement a recursive structure across the set of signal processing algorithms, turning some of the latter into iterated nonlinear transformations.

\section{Audio signal processing (ASP)}

Audio-rate signal transformations are expected either to proliferate the in-coming signal (thus incrementing the total sonic energy in the ecosystem) or to smooth out and even to dissi-pate energy. We opted for timedomain over frequency-domain processing methods, mainly for computational load and real-time constraint considerations.

The Machine Milieu system makes uses of various custom implementations of: sampling/resampling methods, asynchronous granulation, waveshaping (nonlinear wave transfer, modeling distortion), cascaded FM (also in feedback configurations), pulse-width modulation (PWM), highpass and low-pass, as well as all-pass and comb filtering, and feedback delay networks (FDN).

It should be noted that multiple
FDN configurations are involved: some are simply to dispatch signals across the set of audio processing methods, in some cases creating recursive paths and contributing to the developing of different layers of sonic transformations across different time spans; while other FDN configurations, instead, are arranged in ways that de facto get closer to what would be called a "reverb unit" (depending on the question of time scales).

Once the Machine Milieu performance is on its way, sounds born from the two Computer Units overlap and merge with those emanating from the environment (ambiance, noise). In addition, the output of one Computer Unit will feed the input of the other, and vice-versa. When working in feedback conditions, the signal processing transformations will effectively feed each other creating all sorts of multiple processing paths, potentially feeding back into itself (depending on the electroacoustic setup and the room). Thus, the recursive design turns them into iterated functions, and iterated nonlinear functions, due to the inherent nonlinearities in the signal processing transformations, and in the circuitry of the analog transducers involved. All of this creates a complex dynamic system, which contributes to 
the range of sonorities emerging in the process.

\section{Control signal generation (CSG)}

Control signals are generated here as real-time mappings and transformations of data streams obtained from the real-time analysis of sonic properties in the input audio signal. As they are generated, they are also applied to runtime variables in the audio signal processing algorithms. While typically working in the subaudio frequency range, here control signals are processed as audio signals, and accordingly, they can be mapped into the audio range and used as modulation signals, resulting in audible spectral modifications.

We parse control signal generation in two main tasks: an analysis step, and a processing step.

\section{Feature-extraction and analysis}

We cannot predict specifically which kind of sonority will be subject to analysis - it may range from "silence," to background noise, or any ambiance phenomena, to musical gestures eventually born from the performance process itself (of which, however, not much can be said beforehand). Therefore, it is not possible to pre-select specific feature-extrac- tion methods and "tune" them for optimal performance with specific kind of input materials. A more comprehensive approach is needed, exploring more generic analysis methods, and submitting the analysis process parameters to controls signals generated in the performance process (CSG feed-back).

More fundamentally, we do not presume "feature-extraction" methods can "pick up" or "track" any information in the sound environment: what we do instead is to leverage the energies present in the environment (however patterned, or randomly scattered they might be, in time and space) to shape various low-frequency signals used to drive audio signal processing transformations. This approach leans on a constructivist epistemology [Von Foerster 2007], according to which the general idea of pulling information from the environment is misleading and should be replaced with a notion that information is (to be) shaped, construed, not "extracted." In principle, we should abandon a terminology that implies the objecthood of "data" as information collected in the environment, as properties of a sound event, or of its auditory image. Also misleading would be to say that, based on observed data, the 
computer will or may manifest a kind of adaptive behavior: adaptation implies the predefinition of optimal target results, and that is not our task.

The main sonic features considered include loudness, density, brightness, noisiness, and roughness. Overall, these are sonic features that can be loosely referred to as perceptual criteria.

Density is an unusual descriptor. We understand it in terms of root mean square (RMS) values calculated over extended signal segments (in the order of few to several seconds), eventually correlated with peak envelope tracking (attack transients) or other statistical analysis ("amount of attack transients" in a given time frame). In that sense, density means more than anything else a "level of activity," or "busyness." Note that density and loudness can be considered as perceptual correlates: the two may be descriptive of the same feature (energy expense in time), but at different time scales. We often derive control signals based on RMS estimates of loudness and density at multiple time scales.

Brightness and noisiness are calculated via original algorithms operating in the time domain (adaptive equalpower crossover cutoff; adaptive zero- crossing rate differentiation). Another strategy is via averaged responses of large-width band-pass filters, allowing to compare energy levels (loudness) across different spectral regions, and eventually correlating patterns between regions. Roughness estimation uses envelope tracking through instantaneous amplitude, calculated via analytic signals and differentiation (transient detection). Depending on sonic complexity, brightness, noisiness, and roughness may be perceptually correlated, and roughness may also correlate with density estimations.

\section{Mapping and processing}

Feature-extraction methods provide us with streams of source data, from which multiple control signals can be shaped. Various mapping operations can then be adopted. In broad terms, we can distinguish between linear and nonlinear mapping functions of the source data, and between direct or inversely proportional mapping functions.

Whenever possible, a mapping strategy of "one-to-many" appears especially valuable: a single stream of source data is turned into several control signals. Sometimes (very rarely) it is useful to resort to a "many- 
to-one" strategy, doing higher-order statistics of multiple data streams and ending up in a single, generic descriptor, integrating the various source data.

Sequences of mapped values are then also submitted to signal processing (simple filters, delay units, etc.). We call this "control signal processing." The low-frequency signals thus generated are to modulate the control variables in the audio signal processing algorithms. Thus, they become vectors of dynamical behavior, allowing for different developments across shorter and longer time spans, and resulting in timbre variations (signal level transformations) and larger structural articulation (musical gestures).

\section{Musical agency based on site-specific sound}

Because they are generated as a function of the total sound in the performance space, control signals loop back onto themselves through the space, affecting their subsequent unfolding. Also, some of the featureextraction parameters (e.g., window size in RMS estimation, or filter bandwidth, etc.) are themselves driven by controls signals (in a strategy of "adaptive filtering"). Such circum- stances, stem from the recursive design of the ecosystemic process. They represent feed-back mechanisms at control signal level, and give rise to second, or higher-order emergent patterns in the resulting sounding activity.

The latter annotation may be taken to clarify that, in the approach taken here, the real-time and real-space (site-specific) generation of control signals should be acknowledged a crucial role: it provides the potential for the unsupervised articulation of sound and music at performance time. By resorting to higher-order analysis and statistics of lower-level data, Machine Milieu reveals a kind of situated and autonomous agency capable of bringing forth a sense of consistent and oriented process in sound.

From the standpoint of a single Computer Unit, the total sound at any time originates both from its process as well as from the companion Computer Unit - from other sources in performance space, if that is not acoustically dry or idle. Also, the sound captured by the microphones and input to the computer is never identical with the sound delivered by the loudspeakers, because the microphones will also capture all sorts of 
phase (de)correlated acoustical reflections in the performance space. Measuring the differences between signals at the digital to analog (output) and analog to digital (input) converters is a way to track down the contribution of the single Computer Unit from the total sound, and to capture what is acoustically added (or subtracted) by the local environment. Complex dynamics of Selves and nonSelves can then be established, which is after all the core job of the CSG methods involved.

\section{Rethinking networked computing musical agencies}

Densely connected network systems have long been investigated in the context of algorithmically oriented performance practices (e.g., The Hub [Trayle 1991], and the early League of Automatic Music Composers [Bishoff et al. 1978], not to mention today's live coding practices). Haworth [2014] discusses the ecosystemic and technical structure in networked performance ensembles such as The Hub, where the connective medium is typically provided by formal protocols of music data and their transfer along digital channels (MIDI, OSC, if not the stream of digital samples itself). Knotts [2015] discusses the "distribution of power" patterns implicit in networked performance, comparing them with political models.

In the Machine Milieu project, the main sites of agency are integrated as components of a sounding ecosystem: their individual agency, as well as their collective interdependencies, remain under the spell of the permanent mechanical (acoustical) mediation of the local environment. The connections are not through digital nodes and terminals, but rather along the lines of acoustical propagation in the air (diffused via electroacoustic transducers, whose nonlinearity also adds to the contingent materiality of the ensemble). We sympathize with Tim Ingold's critique of the widespread notion of "network" [see Ingold 2011], although we do not feel necessary in the present paper to replace "network" for "meshwork," as Ingold does in his philosophy of anthropology.

A related question can be raised, as to what exactly is meant by "computing" in such a hybrid context. Assemblages of densely interconnected human, electro-mechanical, and digital agencies make it difficult to tell where precisely computations take 
place, particularly when made dependent on the specific physical space [Di Scipio 2015]. In Machine Milieu, computer operations do not necessarily represent the most decisive factor, as far the sounding results are concerned: no symbolic representation, no short- or long-term patterning is formalized in the computer. Yet, can we not say sound and music are being somehow computed, presumably by the overall ecosystemic agency set at work? No syntactical rules are declared; still, a sense of consistent interplay of affordances and limitations, a sense of structure, characterizes the complete performance ecosystem in its real-time and real-space process. The situatedness and contextual dependency of computing resources foster a view of "algorithms" as agencies foreign to an abstract and "immaterial" ontology [Rutz 2016a]. Some sound artists today seem to take a quite radical approach to the materiality of computation [Jordan 2015]. In a larger view, a notion of ecosystemic agency would also stress the distributed and hybrid structure of computation.

\section{Modes of performances}

We consider three distinct modes of
Machine Milieu performance: automatic, participated, and conducted.

Automatic performances means performers shift aside, or refrain from being part of the ecosystem process, letting the network of signal interactions proceed unsupervised. In that case, the two Computer Units are interfering with each other, while also changing their process based on sounds they have delivered in the room at earlier stages.

It is wrong to say that such a performance does entirely without any human intervention. Listening carefully to the room's sounding character, setting the initial conditions to the overall technical process (fine-tuning of variables, placement of microphones and speakers, and more) - all of that is crucial. However, once that is fixed, performers join the audience and hear the growth and development of sonic materials, at least as long as satisfying behaviors seem to arise. When the potential inherent to the particular working conditions seems exhausted, the performers will intervene to alter the working conditions. In rehearsal, we often work like this a few times, trying to grasp the peculiarities of the autonomic process as different from a performance involving the participation of human agents. 
Participated performance means becoming active in the ecosystem. Performers have the following options: (1) they may operate directly on the ASP and CSG variables, manually adjusting numerical values in the signal processing algorithms (via a computer graphic user interface or an external controller); (2) they may reconfigure the mapping and the dispatching of control signals; or, (3) they may act directly on microphones and loudspeakers, changing their position or otherwise modifying their functionality. In some instances, we also utilized small resonators - "found objects," such as carton pipes or boxes, or even hands and mouth - to create a smaller acoustical niches around the microphones.

For each of these options, there might be several "degrees of participation," depending on how responsive and active a performer is in the ecosystemic process. In the Machine Milieu sessions we have had so far, this was experimented by taking different improvisational approaches. Improvisation is very often used when performing with feedback systems [Bowers 2002, Green 2013, Sanfilippo \& Valle 2015], in fact improvisation itself can be said to be intrinsically based on feedback, where current actions are mostly determined by listening and promptly reacting to whatever results from earlier actions. Yet, a generic notion of improvisation may not help to qualify the human performer as a site of agency in its strict interrelationship with nonhuman (algorithmic and environmental) agencies. However improvisational, participated performance is mostly an ongoing negotiation of one's role in the ecosystem process: one takes part in a complex web of continuing exchanges. In our practice, this often translates into an attempt to stabilize the dynamical process for a duration, to support prolonged textures rich in sonic micro-variations. In systemic terms, this is like introducing a form of negative feedback and may represent a challenging task to pursue, given the enormous amount of collateral working conditions and possible sources of perturbations. However "free" and improvisatory, and however "discreet," human actions and goals are constantly put into question by the innumerable, subtle and often unseizable interdependencies among the ecosystem components.

Conducted performance consist of more definite and frequent actions; performers might have a more sig- 
nificant impact on the ecosystemic dynamics, and can eventually pursue more gestural and dramatic developments. In a sense, that opens to more typical musical conducts, where performers take the lead over the available resources and the overall ecosystemic dynamics. They could be said to be "playing." More precisely, they are trying to instrumentalize the performance ecosystem, forcing it towards wanted, specific results.

In actuality, a complete instrumentalization of the technical infrastructure remains out of the question, provided that the structural coupling of the parts (computers, electroacoustic equipment, and performance space) do not only provide affordances but also imposes limitations. Not everything is possible. Limitations may be particularly evident when performers force the process (either inadvertently or purposefully) to operate close to their boundary conditions. This is especially when, perhaps in an approach of "radical improvisation," performers push the process to its limits. In which case, the interplay of affordances and limitations may give special sonorous evidence to the inherent system dynamics.

Paradoxically, such circumstances may be revealing of what we could other-wise call musical form: a delimited field of forces, within whose limits systemic consistency is preserved.

In actual Machine Milieu performance sessions, things are usually more nuanced and coupled than illustrated with the sharp, three-fold classification provided here. Also, the classification does not imply any hierarchy of performance modalities. It only illustrates possibly useful ways to practice and investigate the porous boundaries between the environmental agency that could be acknowledged to our performance ecosystem, and the small and yet significant margin of maneuver afforded to intentional human behavior. What remains crucial is the notion that all agencies involved are taken in a flow of ongoing exchanges or mutual determinations, which cannot be fully characterized in themselves as separate from others.

Final remarks and research implications

By designing it a hybrid (digital, analog and mechanical) sound-generating infrastructure, and by experimenting with the complex network of interdependencies of its compo-nents, we conceive of Machine Milieu as a 
workshop for performers and listeners to ponder questions of con-text awareness, ecosystemic dynam-ics, materiality of algorithms in daily life, and questions of autonomy and agentivity. However varied, such issues converge into the quest for a definition of agency in overly technologized music environments.

Based on our efforts, and in consideration of research work in ecosystem theory and system ecology [Jørgensen \& Müller 2000], we would say that a viable definition of ecosystemic agency is better delineated by rethinking the notion of interactivity (nowadays frequently charged with severe misunderstandings) in the light of the more encompassing notion of structural coupling, a concept rooted in general system theory, and more apt to connote the ways by which living systems deal with the space they dwell in [Maturana \& Varela 1980].

Indeed, definitions of liveness in live electronic music performance [Emmerson 2013, Sanden 2013] may take advantage of a closer examination of the structural coupling inherent to hybrid assemblages such as computer and electroacoustic music performance infrastructures. We think that the sheer presence of human performers operating in and across an overly technologized playground is, in and of itself, insufficient to characterize liveness in such contexts. We rather suggest that liveness is found in the way machines, performers, and physical environments are creatively coupled and made to co-evolve. This view may harmonize questions of liveness, as tackled in performance studies, with broader theoretical perspectives today emphasizing the environmentalization of agency [Clarke \& Hansen 2009, Hörl 2013] and the materiality of the digital [Parisi 2013].

The sound-mediated and distributed agency illustrated by the Machine Milieu performance ecosystem is probably an example of what philosophers Hans-Jörg Rheinberger and Michael Schwab would call an "experimental system," applied in the context of practice-based artistic research [Schwab 2013, Crispin \& Gilmore 2014]. For us, it provides a platform for under-standing musical and sonic creativity as an emergent and indeed ecosystemic phenomenon.

\section{References}

Ashby, W. (1958). "Requisite variety and its implications for the control of com- 
plex systems", Cybernetica 1, pp. 83-99. Barandiaran X. and A. Moreno (2006). "On what makes certain dynamical systems cognitive: A minimally cognitive organization program", Adaptive Behavior 14(2), pp. 171-185. Bateson, G. (1972). Steps to an Ecology of Mind. Chicago: University of Chicago Press.

Benkirane R. et. al. (2002), La complexité. Vertiges et promesses. Le Pommier. Bischoff J., R. Gold, and J. Horton (1978). "Music for an interactive network of microcomputers", Computer Music Journal 2 (3), pp. 24-29. Bowers, J. (2002). "Improvising machines: Ethnographically informed design for improvised electroacoustic music", ARiADATexts 4.

Bown O. and A. Martin (2012). "Autonomy in music generating systems", Musical Metacreation. AAAI Technical Report.

Clarke B. and M.B. Hansen (eds.) (2009). Emergence and Embodiment: New essays on second-order systems theory, Duke University Press.

Crispin D. and B. Gilmore (eds.) (2014). Artistic Experimentation in Music. An Anthology, Ghent: Orpheus Institute. Di Scipio, A. (2003). "Sound is the interface: from 'interactive' to 'ecosystemic' signal processing", Organised Sound 8(3), pp. 269-277.
Id. (2008). "Emergence du son, son d'emergence: Essai d'épistémologie expérimentale par un compositeur", Intellectica, 48/49, pp. 221-249. Id. (2011). “Listening to yourself through the otherself: on Background Noise Study and other works", Organised Sound 16(2), pp. 97-108.

Id. (2015). "The place and meaning of computing in a sound relationship of man, machines, and environment," Array, pp. 37-52.

Id. Les conditions du 'vivant' dans la performance live electronics. Une perspective écosystémique du son et de la musique (forthcoming, EDESTA, Université Paris VIII).

Emmerson, S. (2013). Living electronic music. Ashgate Publishing.

Etxeberria A., J.J. Merelo, and A. Moreno (1994). "Studying organisms with basic cognitive capacities in artificial worlds" Cognitiva 3(2), pp. 203-218. Green, G. (2013). User serviceable parts: Practice, technology, sociality and method in live electronic musicking. Ph.D. dissertation, City University London.

Haworth, C. (2014). "Ecosystem or Technical System? TechnologicallyMediated Performance and the Music of The Hub", Proceedings of the Electroacoustic Music Studies Network. Hörl, E. (2013). “A Thousand Ecologies: 
The Process of Cyberneticization and General Ecology", in: D. Diederichsen and A. Franke (Eds.), The Whole Earth. California and the Disappearance of the Outside, Sternberg Press. Ingold, T. (2011). Being alive: Essays on movement, knowledge and description, Taylor \& Francis.

Jordan, R. (2015). “DIY electronics. Revealing the material system of computation", Leonardo Music Journal 25. Jørgensen S.E. and F. Müller (2000), Handbook of Ecosystem Theories and Management, Lewis Publishers. Knotts, S. (2015). “Changing Music's Constitution. Network Music and Radical Democratization", Leonardo Music Journal 25.

Maturana H. and F.Varela (1980). Autopoiesis and Cognition. The realization of the living, Reidel.

Mitchell, M. (2009). Complexity: A guided tour. Oxford University Press. Parisi, L. (2013). Contagious architecture: computation, aesthetics, and space, MIT Press.

Rutz, H.H. (2016a). "Making a Space of Algorithmicity", Proceedings of 4th Conference on Computation Communication Aesthetics and $X$.

Id. (2016b). "Agency and algorithms", Journal of Science and Technology of the Arts CITAR 8(1), pp. 73-83.

Sanden, P. (2013). Liveness in Modern
Music: Musicians, Technology, and the Perception of Performance. Routledge. Sanfilippo, D. (2013). "Turning perturbation into emergent sound, and sound into perturbation", Interference 3. Idem (2017). "Time-variant infrastructures and dynamical adaptivity for higher degrees of complexity in autonomous music feedback systems: the Order from noise (2017) project", Musica/Tecnologia 11-12, pp. 121-131. Sanfilippo, D. and A. Valle (2013). "Feedback systems: An analytical framework," Computer Music Journal 37(2), pp. 12-27.

Schwab M. (ed.) (2013). Experimental Systems. Future Knowledge in Artistic Research, Ghent: Orpheus Institute. Trayle, M. (1991). "Nature, networks, chamber music", Leonardo Music Journal 1, pp. 51-53.

Von Foerster, H. (2007). Understanding understanding: Essays on Cybernetics and Cognition. Springer Science \& Business Media.

Waters, S. (2007). "Performance Ecosystems: Ecological approaches to musical interaction", Proceedings of the Electroacoustic Music Studies Network. Id. (2013). "Touching at a distance: resistance, tactility, proxemics and the development of a hybrid virtual/physical performance system", in: Contemporary Music Review 32(2/3), pp. 119-134. 